\title{
Comprehensive Geriatric Assessment from a Nursing Perspective
}

\author{
Lina Spirgiene and Louise Brent
}

As the incidence of fragility fractures continues to rise, healthcare professionals will encounter patients with fractures in a variety of clinical settings such as falls clinics, intermediate care services and acute medical wards. Older people with fragility fractures are a diverse group, and their care needs are complex. Although some have comparatively few health problems, many have a series of interconnected illnesses and psychological and social problems requiring a range of therapeutic interventions. The primary focus of care is to meet the needs of the older person following skeletal trauma throughout their care pathway and ensure that they receive the same high standard of specialist care within orthopaedic services as they would within a setting specialising in the care of older people. The central philosophy should be holistic care using a person-centred approach that brings the various aspects of specialist care together.

'Geriatric syndrome' is a term often used to refer to common health problems in older adults that do not fit into distinct organ-specific disease categories and that have multifactorial causes; this includes problems such as frailty, cognitive impairment, delirium, incontinence, malnutrition, falls, gait disorders, pressure ulcers, sleep disorders, sensory deficits, fatigue and dizziness. These are common in older adults and can have a major impact on quality of life (QoL) and disability [1]. Geriatric syndromes can best be identified by a comprehensive multidisciplinary

\footnotetext{
L. Spirgiene, R.N., Ph.D. ( $\bowtie)$

Medical Academy, Faculty of Nursing, Department of Nursing and Care,

Lithuanian University of Health Sciences, Kaunas, Lithuania

Nursing Coordination Department, Hospital of Lithuanian University of Health Sciences Kauno Klinikos, Kaunas, Lithuania

L. Brent

National Office of Clinical Audit, St Stephen's Green, Dublin 2, Ireland

e-mail: louisebrent@ noca.ie 
geriatric assessment so that they can inform planning appropriate interventions within a team approach.

Problems that relate to ageing such as functional impairment and dementia are common and often unrecognised or inadequately addressed. Identifying problems specific to ageing so that interventions can be tailored to meet the specific needs of patients with fragility fractures requires a detailed and comprehensive assessment that can help clinicians manage these conditions and prevent or delay their complications. This needs to be conducted by the whole multidisciplinary team so that the skills of each team member can contribute to building a picture of the patient's needs. Nursing assessment is a significant part of this whole. A term often used in relation to the assessment of older people with medical needs is comprehensive geriatric assessment (CGA). This approach is designed to accommodate the multidisciplinary approach that is so important in the care of the older person in any setting, and the role of nursing assessment within this is central to planning effective interventions to resolve nursing-focused problems.

The aim of this chapter is to explore the nature of comprehensive geriatric assessment (CGA) for the patient with a fragility fracture and discuss how this can be applied to nursing assessment and care.

\subsection{Learning Outcomes}

At the end of the chapter, and following further study, the nurse will be able to:

- Explain the principles of comprehensive geriatric assessment (CGA) from a nursing perspective

- Identify how the CGA process applies to the whole multidisciplinary team

- Discuss the nursing contribution to comprehensive assessment of the older person with fragility fracture.

\subsection{The Concept of CGA}

Assessment involves collecting information about a person's circumstances and needs and making sense of that information to help in decision-making about what support, treatment and care is needed; it should be timely and comprehensive [2]. The assessment of older people differs from standard medical review in three ways: (1) it focuses on older people with complex problems; (2) it emphasises functional status and quality of life; and (3) it takes advantage of an interdisciplinary team. Getting to know people, their strengths and needs is an important first step in effective care of older people [3], and this has been reflected in the APIE (assessment, planning, implementation and evaluation) approach to nursing for more than 50 years. It is recognised that older people receiving nursing care should have the same comprehensive assessment and risk identification to facilitate the identification of individual needs, care planning and 
identification of risks that might impact on optimal care outcomes and inform effective discharge planning [4].

Comprehensive geriatric assessment (CGA) is a multidimensional, interdisciplinary process designed to detect and assess frailty [5], to determine a frail older person's medical conditions, mental health, functional capacity and social circumstances [6] and to identify their care and treatment needs. There is strong evidence that CGA can reduce mortality, increase the number of patients who can return home after hospitalisation and reduce length of stay [7]. The purpose is to plan and carry out a holistic plan for treatment, rehabilitation, support and long-term followup [8]. CGA is part of an integrated approach to assessment based on the following principles [9]:

- The process is person-centred

- The older person's capacity to participate in the process voluntarily must be assessed; if capacity does not exist, there should be a system in place that considers their needs within an ethical framework

- Links between social and healthcare need to be made so that older people who need CGA receive it efficiently in a way that considers their degree of need in timely manner

- Assessments are carried out to a reliable standard within and across multidisciplinary teams.

Models of CGA have evolved in different healthcare settings and to meet differing needs, although not specifically in relation to the management of the patient with fragility fracture. The skill, at the heart of orthogeriatric care, when working with patients with fragility fracture is developing a comprehensive picture of the potential impact of co-morbidities and functional capacity to try to predict their potential impact on the patient's recovery and rehabilitation following the fracture [6] and for this knowledge to direct healthcare practice. There is limited discussion of the role of nurses in the process of CGA as its development has been led by geriatricians. There is good reason, however, for nurses to begin to incorporate CGA into their own practice with patients with fragility fractures because of its potential to provide a clear overview of the patient's healthcare and nursing needs. This may mean that nurses will need to adapt the process to reflect the purpose of nursing and to avoid an overly medicalised approach to care.

Common to CGA are several key features that have been attributed with its effectiveness and can be applied to the patient with fragility fracture, including [10]:

- Co-ordinated multidisciplinary assessment, so that each member of the team can contribute expertise; the team is commonly made up of a geriatrician/physician, nurse and therapists but can involve other health professionals depending on need

- One team member 'in charge' as the co-ordinator or 'case manager' of the process

- Geriatric medicine expertise, so that the medical management of the patient's health problems can lead multidisciplinary interventions 
- Identification of medical, physical, social and psychological problems, so that a comprehensive picture can be obtained and the impact of each of these understood

- Leading to the formation of a plan of care that includes appropriate rehabilitation.

The first step in CGA is to identify those individuals who are likely to benefit from this process as well as the orthogeriatric team approach. Decision-making criteria used to identify patients could include:

- The age of the person and the way in which their ageing process is manifested, e.g. frailty

- Existing medical conditions that are likely to impact on care, recovery and outcomes

- The presence of psychosocial disorders such as depression or social isolation

- Specific 'geriatric syndrome' conditions such as dementia, falls, functional disability, sarcopenia and frailty

- Previous or predicted high need for healthcare use

- Recent change in living situation, e.g. from independent living to assisted living, nursing home or in-home caregivers

- Major illnesses such as those requiring hospitalisation (such as a fracture) or increased need for home care resources to manage medical and functional needs.

The CGA process should begin on admission, encompassing emergency care; but it is not a one-off process, so should continue throughout the care process with constant review and evaluation. To facilitate recording and sharing of assessment findings, the multidisciplinary team should share documentation that includes a proforma to help clinicians to follow the process. In an ideal world, the same process should follow the older person after discharge to home care and other communitybased care facilities.

Performing a comprehensive assessment is an ambitious undertaking and is often more complex than it may seem (Box 4.1), as older people often struggle to recall their past medical history and cognitive impairment can make it difficult for them to answer questions. Resolving this issue involves skilled communication with the patient and collaboration with family and other people who know the patient well to help with history taking.

Box 4.1: Areas of Assessment That Team Members May Choose to Assess Depending on Patient Needs

- Current symptoms and illnesses and their functional impact

- Current medications, their indications and effects

- Relevant past illnesses 
- Recent and impending life changes

- Objective measure of overall personal and social functionality

- Current and future living environment and its appropriateness to function and prognosis

- Family situation and availability

- Current caregiver network including its deficiencies and potential

- Objective measure of cognitive status

- Objective assessment of mobility and balance

- Rehabilitative status and prognosis if ill or disabled

- Current emotional health and substance abuse

- Nutritional status and needs

- Disease risk factors, screening status and health promotion activities

- Services required and received

Conceptually, CGA involves several processes of care that are shared over several members of the assessment team (Box 4.2).

\section{Box 4.2: Elements of Comprehensive Geriatric Care}

- Data gathering

- Biopsychosocial assessment

- Discussions among the team

- Patient and/or caregiver as a member of the team involvement

- Treatment and nursing plan development, with the patient and/or caregiver

- Implementation of the treatment and nursing plan

- Monitoring response to the treatment and nursing plan

- Revising the treatment and nursing plan

CGA, undertaken by multiple personnel over many encounters, is best suited for older people with multiple medical problems and significant functional limitations. An interdisciplinary team, representing medicine, psychiatry, social work, nutrition, physical and occupational therapy and others, should perform a shared detailed assessment, analyse the information, devise a collaborative intervention strategy, initiate treatment and follow up on the patient's progress.

Significantly, older patients are likely to survive admission to hospital and return home if they undergo CGA, while they are inpatients [11], and, if indicated, it should be initiated as soon as possible after admission by a skilled, senior member of the multidisciplinary team and used to identify reversible medical problems, target rehabilitation goals and plan all the components of discharge and post-discharge support needs [12]. 


\subsection{Dimensions of Comprehensive Geriatric Assessment}

Comprehensive assessment involves looking not only at disease states as a standard medical assessment would do, or at functional ability as a standard rehabilitation assessment might do, but at a range of domains. By assessing each of these domains of health, a comprehensive assessment can be made, and the full biopsychosocial nature of the individual's problems can be identified. This process can be supported by using standardised scales and tools, or full formal assessment schemes such as the 'interrai' assessment tools (www.interrai.org). Using standardised scales can encourage consistent practice, help to ensure safety (e.g. pressure injury risk screening) and enable detection of serial changes, but they can also be time-consuming and clinically constraining. Clinicians undertaking CGA should consider the extent to which standardised approaches are helpful in their setting [12]. Core components of CGA that should be considered during the assessment process are outlined in Table 4.1.

Functional status: Functional status relates to the ability to perform activities necessary or desirable in daily life. It is directly influenced by health conditions, particularly in the context of an older person's environment and social support network. Changes in functional status (e.g. not being able to bathe independently) should prompt further diagnostic evaluation and intervention. Measurement of

Table 4.1 Domains and suggested items for comprehensive geriatric assessment (BGS 2010)

\begin{tabular}{|c|c|}
\hline Domain & Suggested items for assessment \\
\hline $\begin{array}{l}\text { Physical health and medical } \\
\text { conditions }\end{array}$ & $\begin{array}{l}\text { Comorbid conditions and disease severity } \\
\text { Medical review } \\
\text { Nutritional status } \\
\text { Polypharmacy } \\
\text { Urinary continence } \\
\text { Sexual function } \\
\text { Vision/hearing } \\
\text { Dentition }\end{array}$ \\
\hline $\begin{array}{l}\text { Mental health and } \\
\text { psychological status }\end{array}$ & $\begin{array}{l}\text { Cognition } \\
\text { Mood and anxiety } \\
\text { Fears } \\
\text { Goals of care } \\
\text { Advance care preferences } \\
\text { Spirituality }\end{array}$ \\
\hline Functioning & $\begin{array}{l}\text { Functional capacity: core functions such as mobility and } \\
\text { balance, fall risk } \\
\text { Activities of daily living } \\
\text { Life roles that are important to the patient }\end{array}$ \\
\hline Social circumstances & $\begin{array}{l}\text { Social support and networks: } \\
\text { Informal support available from family } \\
\text { Wider network of friends and contacts } \\
\text { Statutory care } \\
\text { Financial concerns and poverty }\end{array}$ \\
\hline Environment & $\begin{array}{l}\text { Living situation: housing, comfort, facilities and safety } \\
\text { Use or potential use of 'telehealth' technology } \\
\text { Transport facilities } \\
\text { Accessibility to local resources }\end{array}$ \\
\hline
\end{tabular}


functional status can be valuable in monitoring response to treatment and can provide prognostic information that assists in long-term care planning. With respect to the impact of functional status on activities of daily living (ADLs), an older person's functional status can be assessed at three levels: (1) basic activities of daily living (BADLs), (2) instrumental or intermediate activities of daily living (IADLs) and (3) advanced activities of daily living (AADLs). BADLs consider self-care tasks which include; bathing, dressing, toileting and maintaining continence, grooming, feeding and transferring. IADLs consider the ability to maintain an independent household which includes shopping for groceries, driving or using public transportation, using the telephone, performing housework, home maintenance, preparing meals, doing laundry, taking medication and handling finances.

In addition to considering ADLs, gait speed alone predicts functional decline and early mortality in older adults. Assessment of gait speed is the domain of the physiotherapist within the team and may identify patients who need further evaluation, such as those at increased risk of falls. Assessing gait speed may also help identify frail patients who might not benefit from treatment of chronic asymptomatic diseases such as hypertension. For example, elevated blood pressure in individuals age 65 and older is associated with increased mortality only in individuals with a walking speed $\geq 0.8 \mathrm{~m} / \mathrm{s}$ (measured over $6 \mathrm{~m}$ or 20 feet) [13].

Falls: Approximately one-third of community-dwelling people over 65 years and one-half of those over 80 years of age fall each year [14]. Those who have fallen or have a gait or balance problem are at higher risk of having a subsequent fall and losing independence. An assessment of fall risk should be integrated into the history and physical examination of all older patients (Chap. 3).

Cognition: The incidence of dementia and delirium increase with age, particularly among those over 85 years; yet many older people with cognitive impairment remain undiagnosed. The value of making an early diagnosis includes the possibility of uncovering treatable conditions. The evaluation of cognitive function can include a thorough history, brief cognition screening, a detailed mental status examination, neuropsychological testing and other tests to evaluate medical conditions that may contribute to cognitive impairment (Chap. 9).

Mood: Depressive illness in older people is a serious health concern leading to unnecessary suffering, impaired functional status, increased mortality and excessive use of healthcare resources (Chap. 9). Depression in later life remains underdiagnosed and inadequately treated. Depression in older adults may present atypically and may be masked in patients with cognitive impairment. Screening is easily administered and likely to identify patients at risk if both of the folowing questions are answered affirmatively:

1. 'During the past month, have you been bothered by feeling down, depressed, or hopeless?'

2. 'During the past month, have you been bothered by little interest or pleasure in doing things?'

Polypharmacy: Older people are often prescribed multiple medications by different healthcare providers, placing them at increased risk of drug interactions and 
adverse medication events. The clinician should review medications at each visit. The best method of detecting potential problems with polypharmacy is to have patients provide all medications (prescription and non-prescription) in their packaging. Alternatively, practitioners should contact the patient's primary care physician, particularly if the patient cannot remember their medications. As some health systems have moved towards electronic health records and electronic prescribing, the possibility of detecting potential medication errors and interactions has increased. Older people should also be asked about alternative medical therapies by asking about herbal medicine use with the question: 'What prescription medications, over the counter medicines, vitamins, herbs, or supplements do you use?'

Social and financial support: The existence of a strong social support network in an older person's life can frequently be the determining factor of whether the patient can remain at home or needs placement in a residential care setting. A brief screen of social support includes taking a social history and determining who would be available to help if they become ill. Early identification of problems with social support can help planning and timely development of resource referrals. For patients with functional impairment, the practitioner should ascertain who the person has available to help with ADLs. It is also important to assess the financial situation of a functionally impaired older adult; some may qualify for state or local benefits, depending upon their income. Occasionally, there are other benefits such as longterm care insurance or veteran's benefits that can help in paying for caregivers and prevent the need for institutionalisation.

The gathering of information is more complex than it seems [7], particularly collecting accurate baseline information from patients who may have cognitive difficulties, espeically if the environment is noisy such as in the ED or busy trauma unit, in the presence of pain or opioid analgesia use or anaesthesia. In the first few hours following admission, the patient is more likely to recall the history of the injury due to more recent recall, but this period is also very stressful. Collecting detailed and accurate information needs specialised skills in communication and an expert understanding of the process of assessment.

\subsection{Assessment Tools}

Although the amount of potentially important information may seem overwhelming, formal assessment tools and shortcuts can reduce this burden on the clinician performing the initial CGA. A previsit questionnaire sent to the patient or caregiver prior to the initial assessment can be timesaving when there is a need to gather a large amount of information and timing allows, although this is rarely an option when there is an acute admission. Questionnaires can be used to gather information about general history (e.g. past medical history, medications, social history, review of systems), as well as gather information specific to CGA, such as:

- Ability to perform functional tasks and need for assistance

- Fall history

- Urinary and/or faecal incontinence 
- Pain

- Sources of social support, particularly family or friends

- Depressive symptoms

- Vision or hearing difficulties

- Whether the patient has specified a 'lasting power of attorney' for healthcare.

Support staff can be trained to administer screening instruments to both save time and help the clinician to focus on specific disabilities that need more detailed evaluation.

\subsection{Posthospital Discharge}

Key elements of posthospital discharge CGA include targeting criteria to identify vulnerable patients, a programme of multidimensional assessment, comprehensive discharge planning and home follow-up by nurses with specialised geriatric practitioner training who visit patients during hospitalisation and at least twice during the weeks following discharge. This intervention is usually initiated 1-2 days prior to hospital discharge. Like home assessments, post-discharge home visits are supplemented by telephone calls and additional visits by physical therapy, occupational therapy, social work and/or home nursing services when indicated (Chap. 10).

\subsection{Secondary Prevention}

Secondary prevention of fragility fractures (Chap. 3) should be approached in a systematic and coordinated manner, to some extent during the inpatient stay, but continuing after discharge. All patients presenting with fragility fractures should be assessed by an orthogeriatrician or other specialist with respect to their ongoing fracture risk. This may trigger referral to other specialists such as endocrinologists for further investigation. Referrals must also be made to secondary fracture prevention services (Chap. 3) where patients can be reviewed by a fracture prevention practitioner, orthogeriatrician, endocrinologist and dietician. The assessment, diagnosis and referral process can be coordinated by a fracture prevention practitioner and treatment initiated and followed up accordingly. Patients should also be referred, where appropriate, to falls clinics.

\subsection{The CGA Team}

The assessment team varies depending on the service and can include the full range of healthcare professionals working in the multidisciplinary team. In many settings, the CGA process relies on a core team consisting of a medical clinician, nurse, therapist and social worker and, when appropriate, draws upon an extended team including occupational and other therapists, nutritionists, pharmacists, psychiatrists, psychologists, dentists, audiologists, podiatrists and ophthalmologists/optometrists. 
Although these professionals can work in the hospital setting, many are also available in the community. Increasingly, there is a move towards a 'virtual team' concept in which members are included as needed, assessments are conducted at different locations on different days, often using the electronic health record but stored electronically and accessible anywhere, and team communication is completed via telephone or electronically,.

Traditionally, the various components of the process are completed by different members of the team, with considerable variability in the way assessments are conducted and recorded. The medical assessment of older people may be conducted by a physician (usually a geriatrician), nurse practitioner, physiotherapist or physician assistant. The core team (geriatrician, nurse, therapist, social worker) may conduct only brief initial assessments or screening for some dimensions. These may be subsequently augmented with more in-depth assessments by additional professionals; e.g. a dietitian may be needed to assess dietary intake and make recommendations on optimising nutrition, or an audiologist may need to conduct a more extensive assessment of hearing loss and evaluate an older person for a hearing aid.

Because of the 24-h nature of their practice and the wide range of care, nurses are often expected to take a leading role in the care of older people and to coordinate the assessment process. Despite this, the role of the nurse in CGA is ill defined and is not considered in detail in the literature. The potential for nurses, particularly those with advanced assessment skills, to act as a fulcrum for the CGA process is largely untapped. Nursing is already directed by the nursing process: incorporating APIE. Clarke [3] suggests that this traditional view of the nursing process focuses on identifying need deficit and that a more effective philosophy is to assess the resources of older people themselves and jointly plan care alongside the MDT, patients and carers so that as much self-management is retained as possible. Nurses place importance on coming to know a person as an individual through a continuous and ongoing assessment process that will support the rest of the nursing process (planning, implementation and evaluation) and help them to provide effective care. This knowledge can only be achieved by a comprehensive assessment process that incorporates the biological, psychological, social and spiritual dimensions of the person [15].

While the CGA process has not been specifically developed to capture patients' nursing needs, it has the potential to become a holistic multidisciplinary assessment for the whole team and to ensure that the complex needs of patients with fragility fractures are fully met through a continuous process while looking for changes in the patient's condition. The whole team need to work together to further develop this process from a collaborative perspective so that the many different forms of mono-disciplinary assessment processes and associated documentation can be brought together as a single, effective process [4]. New or adapted assessment tools may be required for use by all professionals in the team that can be used to facilitate multidisciplinary and interagency working [16] but also with a view to seamless transfers between primary and secondary care settings. All practitioners should be able to use the information generated during CGA to develop treatment and longterm follow-up plans, arrange for primary care and rehabilitative services, organise and facilitate the intricate process of case management, determine long-term care requirements and optimal placement and make the best use of healthcare resources. 
The assessment process in most units is not perfect, and there is a need to identify ways to both improve the assessment process and demonstrate the value of nursing in this central aspect of care.

\subsection{Summary of Key Points}

- Timely and comprehensive assessment is essential in understanding the needs of older people and ensuring that their needs are met through care and treatment

- CGA is a person-centred, holistic, multidisciplinary process that helps to assess the frail older person so that their medical conditions, mental health, functional capacity and social circumstances can be considered in detail and from which patients with fragility fractures can benefit significantly

- The process should begin on admission and be followed through to post-discharge care in primary and residential care settings: it is not a one-off process but should be subjected to constant review and evaluation

- The CGA process should, as a minimum, consider the domains of physical health and medical conditions, mental health and psychological status, functioning, social circumstances and environment so that MDT care and treatment can be based on the needs generated by these

- Assessment tools need to be developed, or adapted, to meet the needs of this interdisciplinary process and can include existing assessment and screening tools. Interdisciplinary team collaboration will be needed in making this process work in the best interests of patients with fragility fractures.

\subsection{Suggested Further Study}

- Think about how you currently conduct assessment in your place of work-does it fit in with the CGA approach to assessment?

- What skills do you/your team need for you to improve how you assess patients using this approach?

- How might you learn these skills and how would you assess what you have learnt?

- Discuss with other members of the multidisciplinary team within which you work how you might move towards a full team approach to the CGA process and what changes might be needed for this to happen.

\subsection{Self-Assessment}

- Examine the current assessment documentation used in your unit and consider whether it reflects:

- Comprehensiveness

- Patient-centeredness

- Multidisciplinary team working

- Reflect on how this could be adapted and improved. 


\section{References}

1. Inouye SK et al (2007) Geriatric syndromes: clinical, research and policy implications of a core geriatric concept. J Am Geriatr Soc 55(5):780-791

2. Slater P, McCormack B (2005) Determining older people's needs for care by Registered Nurses: the Nursing Needs Assessment Tool. J Adv Nurs 52(6):601-608

3. Clarke CL (2012) Fundamentals of nursing. In: Reed J et al (eds) Nursing older adults. Open University Press, Maidenhead, pp 79-79

4. Langdon R et al (2013) Assessment of the elderly: it's worth covering the risks. J Nurs Manag 21:94-105

5. Smith G, Kydd A (2016) Editorial: Getting care of older people right. The need for appropriate frailty assessment. J Clin Nurs 26:5-6

6. British Geriatric Society (BGS), Comprehensive assessment of the frail older patient. 2010. http://www.bgs.org.uk/good-practice-guides/resources/goodpractice/gpgcgassessment\#

7. Wilson, H. (2017) Pre-operative management. In: Falaschi, P. \& Marsh, D. Orthogeriatrics. Springer: Basel pp 63-79

8. Stuck AE et al (1993) Comprehensive geriatric assessment: a meta-analysis of controlled trials. Lancet 342:1032

9. Devons CA (2002) Comprehensive geriatric assessment: making the most of the aging years. Curr Opin Clin Nutr Metab Care 5:19

10. Ellis $\mathrm{G}$ et al (2011) Comprehensive geriatric assessment for older adults admitted to hospital: meta-analysis of randomised controlled trials. BMJ 343:d6553

11. Oliver D et al (2014) Making our health and care systems fit for an ageing population. Kings Fund, London

12. Welsh TJ et al (2014) Comprehensive geriatric assessment-a guide for the non-specialist. Int J Clin Pract 68(3):290-293

13. Studenski $\mathrm{S}$ et al (2011) Gait speed and survival in older adults. JAMA 305(1):0-58

14. Lord SR et al (2007) Falls in older people: risk factors and strategies for prevention. Cambridge University Press, Cambridge

15. Locsin RC, Purnell MJ (2009) A contemporary nursing process. Springer, New York

16. Folder-Like et al (2013) Development and evidence base of a new efficient assessment instrument for international use by nurses in community settings with older people. Int J Nurs Stud 50:1180-1183

Open Access This chapter is licensed under the terms of the Creative Commons Attribution 4.0 International License (http://creativecommons.org/licenses/by/4.0/), which permits use, sharing, adaptation, distribution and reproduction in any medium or format, as long as you give appropriate credit to the original author(s) and the source, provide a link to the Creative Commons license and indicate if changes were made.

The images or other third party material in this chapter are included in the chapter's Creative Commons license, unless indicated otherwise in a credit line to the material. If material is not included in the chapter's Creative Commons license and your intended use is not permitted by statutory regulation or exceeds the permitted use, you will need to obtain permission directly from the copyright holder. 